\title{
KALMAN FILTER BASED RAILWAY TRACKING FROM MOBILE LIDAR DATA
}

\author{
Yoonseok Jwa and Gunho Sonh* \\ GeoICT Laboratory, Department of Earth and Space Science and Engineering, Lassonde School of Engineering, York University \\ Toronto, Ontario M3J 1P3, Canada - (yjwa, gsohn)@yorku.ca
}

\author{
Commission III, WG V/3
}

KEY WORDS: Railway Track Modeling, Kalman Filtering, Bayesian Decision, Mobile LiDAR Data

\begin{abstract}
:
This study introduces a new method to reconstruct 3D model of railway tracks from a railway corridor scene captured by mobile LiDAR data. The proposed approach starts to approximate the orientation of railway track trajectory from LiDAR point clouds and extract a strip, which direction is orthogonal to the trajectory of railway track. Within the strip, a track region and its track points are detected based on the Bayesian decision process. Once the main track region is localized, rail head points are segmented based on the region growing approach from the detected track points and then initial track models are reconstructed using a third-degree polynomial function. Based on the initial modelling result, a potential track region with varying lengths is dynamically predicted and the model parameters are updated in the Kalman Filter framework. The key aspect is that the proposed approach is able to enhance the efficiency of the railway tracking process by reducing the complexity for detecting track points and reconstructing track models based on the use of the track model previously reconstructed. An evaluation of the proposed method is performed over an urban railway corridor area containing multiple railway track pairs.
\end{abstract}

\section{INTRODUCTION}

A precise and effective maintenance of railway infrastructure should be guaranteed to find a solution for high operating safety and low maintenance costs. For this, the key operation is to perform the evaluation of as-built condition by analyzing track profile and level as well as overall geometry and undulation. 3D modeling of railway tracks can provide significant benefit in precisely monitoring potential risks of railway infrastructure. The track models allow users to: (a) accurately detect wear and deformation of tracks; (b) easily perform collision test and clearance measurement by simulating rail car operating along the entire track route; (c) carry out an immediate response to violations; (d) do cost-effective maintenance by keeping a database of railway systems up to date.

Various techniques related to the railway track model reconstruction from Imagery and LiDAR data have been proposed in literature. The reported algorithms using images which are in $\mathrm{b} / \mathrm{w}$ or color often depend on the following key assumptions to obtain a tractable practical solution to railway scene complexity. (a) As metals such as iron and steel are usually used for the track material, railway tracks are a distinctive object with uniform brightness. The methods normally start to extract supporting cues in detecting tracks based on the evaluation of similarity or homogeneity of image gradients between tracks and the corresponding background. However, the detection methods are sensitive to variation of background and noise caused by shadows and occlusions. (b) Railway tracks are obviously arranged parallel to each other with certain orthogonal distance. This allows us to use simple linear operators with the fixed search space for detecting tracks. However, the applications are limited to cases with the complex distribution of tracks. Especially in the railway switch area, tracks are placed closer together and go in different direction. Based on the key assumptions, many researchers have introduced different algorithms such as knowledge-based track edge detection (Espino and Stanciulescu, 2012), detection rulebased self-adapting algorithm (Beger et al., 2012), spectral estimation and signal-processing based method (Resendiz, 2013) and HOG feature-based track and turnout recognition (Qi et al., 2013).

Compared to the image-based approaches, the LiDAR as an active remote sensing system enables detailed capture of a 3D railway scene with high point density for example in a number of hundreds per square meter. This allows us to readily detect track objects in the 3D space by compensating the weaknesses derived from image-based techniques. By using the massive 3D point clouds, one can refer to literature based on knowledge based track detection and Markov Chain Monte Carlo based track model parameter estimation (Oude Elberink et al., 2013), Kalman filter based track detection (Muhamad et al., 2013), cross-section based template matching (Yang and Fang, 2014). Despite the contributions of the LiDAR data-based studies, there are some limitations affected by the following: (a) geometric constraints of track structures and a priori information of railway scene are used. (b) The methods are sensitive to various factors including point density and scene complexity. This causes the uncertainty in separating track points from terrain points. To overcome the limitation, this study integrated track point detection with track modelling in the Kalman filter framework. It enhances the efficiency of the track modelling process by simultaneously capturing track points along the predicted track models and updating the previous track models.

\footnotetext{
* Corresponding author
} 


\section{METHODOLOGY}

Figure 1 illustrates the overall proposed approach, Kalman filter based railway tracking, for detecting and modelling railway tracks, which is mainly comprised of three steps: (a) preprocessing for determining railway orientation and extracting a local search region, called a strip, from raw mobile LiDAR data. (b) Railway track localization for clustering track components and reconstructing initial track models. (c) Railway tracking by detection for simultaneously capturing railway points and modelling railway tracks in the Kalman filter framework. Finally, the track geometric information is calculated as byproducts which are very important factors in the precise risk monitoring of the railway tracks.

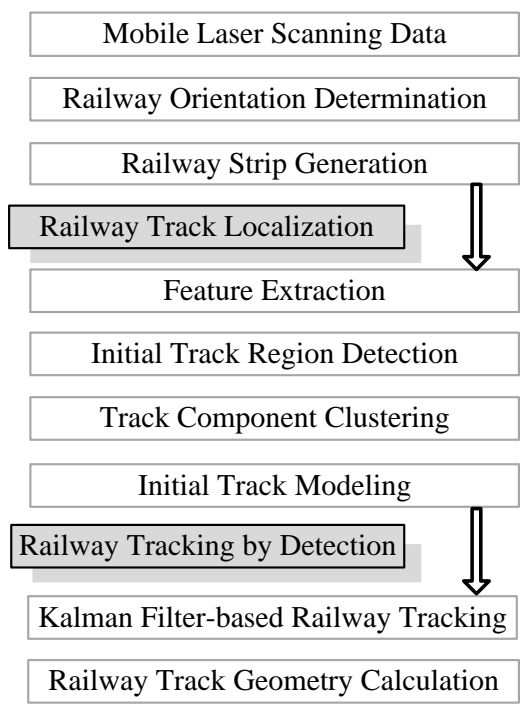

Figure 1. Overall workflow of proposed methods for railway tracking in the Kalman Filter framework.

\subsection{Railway Orientation Determination}

In this study, a strip, is used as a data processing unit space for detecting initial track regions and generating initial track models. As the strip is extracted in the orthogonal direction to the orientation of railway track trajectory, the orientation $\vec{T}(\theta)$ is required to be calculated in advance. For this, a line equation with implicit form, $L(\theta, \rho): \rho=x \cos \theta+y \sin \theta$, is used and its parameters are estimated based on the weighted least squares minimization, thereby using the angle as the railway orientation. Where, $\theta$ and $\rho$ are the angle of line's normal vector and Xaxis and the distance between the line and the origin respectively. The first step is to partition point data into a fixed grid space to effectively deal with the massive point clouds (Cho et al., 2004). Next, center points are calculated using member points belonging to each grid space. Lastly, the line parameters are estimated using the center points and their point densities of each grid space. As the point density more or less consistently decreases if points are far from a main track region, it can be considered as a weight value in the estimation process of the railway trajectory vector. The main reason is that the LiDAR system is a line-of-sight system with a certain angular resolution and operated along the main track region. Figure 2 shows the approximated $2 \mathrm{D}$ vector of railway track trajectory $\vec{T}(\theta)$ and the derived initial strip expressed by a rectangular box.

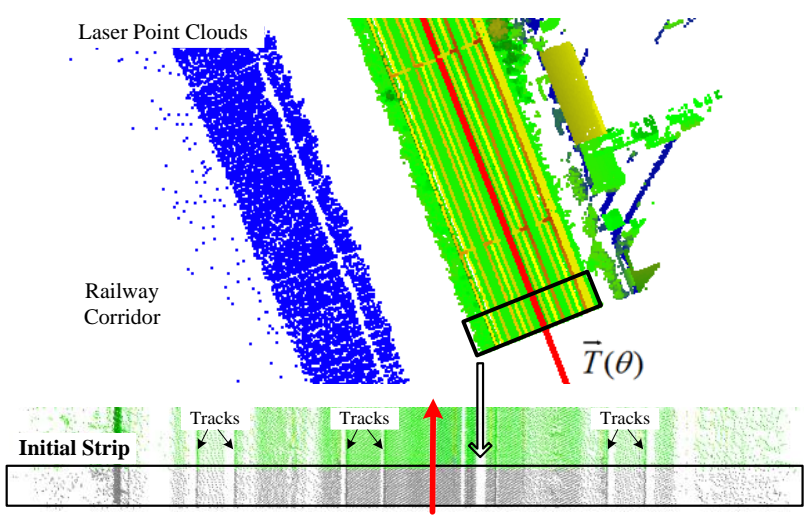

Figure 2. Determination of railway track trajectory, $\vec{T}(\theta)$, (red colored line) and extraction of the initial strip (a rectangle).

\subsection{Railway Track Localization}

The extracted strip contains various objects such as terrain, vegetation, platform, power line as well as tracks. In addition to that, the track is a small and thin object and directly contacts with the ground even the some part of rail foot is integrated with the ground as shown in Figure 3. This causes the scene complexity and uncertainty for detecting the tracks and separating them from the ground. To deal with the uncertainty, in this study, we turn to the Bayesian view in which the uncertainty is quantified by the corresponding probabilities. As a single-track railway consists of two tracks, it is firstly required to detect track zones including the track conjugate pairs in the initial strip. For this, a supervised classification as a machine learning technique is adopted by using a set of features which represents unique characteristics in describing the track zone. Additionally, we also focus on only detecting rail head and web which are considered to comprise a full track object to avoid the scene complexity.

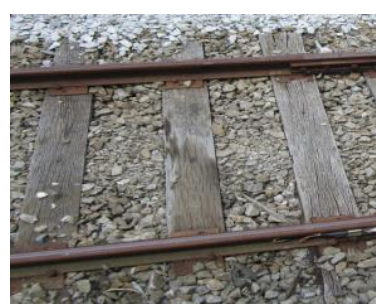

(a)

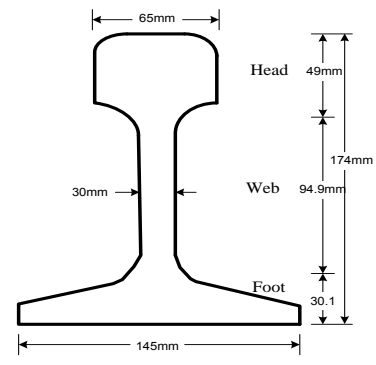

(b)

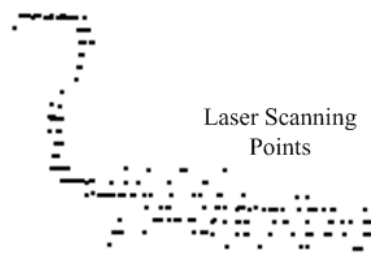

(c)

Figure 3. Railway formation: (a) track region image, (b) KS-60 railway track standard (KRRI, Korean Rail Research Institute), and (c) laser scanning points representing the track. 
2.2.1 Feature Extraction: Three types of features are proposed as follows: mixing coefficients of GMM (Gaussian Mixture Model), HOG (Histogram of Oriented Gradients), and contextual features. The proposed features for detecting a track can be considered as a scale- and orientation-invariant feature. However, the GMM feature can be affected by the scale that is the size of search window. To avoid the problem, the size of the moving window for this study is fixed using $1.5 \mathrm{~m} \times 1.5 \mathrm{~m}$, which is derived from a priori knowledge on the orthogonal distance between tracks. Thus, the features are measured by discretely moving the search window from left to right side in the strip.

First, in the GMM feature extraction, a local search space located around the track is composed of two main segments, a higher one for the track and lower one for the ground in the cross-section view as shown in Figure 3(c). It is assumed that the distribution of points belonging to the search space can be expressed by a mixture of two Gaussian distributions $N(\mu, \Sigma)$ with expectation $\mu$ and variance $\Sigma$. The Gaussian mixture model $p(x)$ is formulated as:

$$
p(x)=\sum_{i=1}^{2} \pi_{i} N\left(x \mid \mu_{i}, \Sigma_{i}\right), \quad \sum_{i=1}^{2} \pi_{i}=1
$$

where, $\pi_{i}$ is called mixing coefficients and used to normalize the Gaussian densities which sum to 1 and $\pi_{i} \geq 0$ for all $i$.

Thus, as a track standard has a certain dimension (Figure 3(b)), we can expect that the difference of mixing coefficients becomes almost constant value in the moving window containing tracks. The GMM parameters including the mixing coefficients are iteratively optimized in the EM (ExpectationMaximization) framework (Ozerov et al., 2011).

Second, HOG is known as a robust local feature descriptor with scale and rotation invariance in the image domain introduced by Dalal and Triggs (2005) (Qi et al., 2013). To extract the HOG feature from point clouds, the first step is to divide the moving window into a regular grid space. Then, track candidate points are detected based on GMM-EM classifier in the each grid. Under the assumption that a track is a linear object in the local search space, the linear vector with its variance is generated using the track candidate points for each grid in the horizontal plane. Then, the orientations of the vectors $\theta_{i}=\left\{0^{\circ} \leq \theta_{i} \leq 180^{\circ}\right\}$ are quantized into eight directions $\left\{\theta_{i}: i=1, \ldots, 8\right\}$ based on CLF (Compass Line Filter) suggested by Sohn et al. (2008). Lastly, those orientation vectors are accumulated by using the corresponding variances as a weight and the normalized histogram with range from 0 to 1 is generated as a HOG feature. Therefore, we believe that the one of eight vectors has a dominant value in the HOG feature histogram for the track zone. Third, a railway structure is constituted to be a pair of single tracks and parallel to each other. Based on the prior knowledge, a pair-wise geometric information comprising contextual features, OD (orthogonal distance between tacks) and IA (inner angle between the OD and the tack vector), can be extracted. As a result, the contextual features become the strong evidences of track existence and are expected to compensate the uncertainty related to the geometric similarity of objects' appearance in detecting tracks.
2.2.2 Track Region Detection: This step aims to detect track candidate zones and select a zone as a main track region in the Bayesian perspective. For this, we adopted a loss function (Eq. (2)) which is used to make as few misclassifications as possible by minimizing its expected loss (Bishop, 2006). We assign points belonging to the search window to one of two classes, a track region and non-track region based the cost function minimizing the total loss caused by the misclassification.

$$
R\left(\alpha_{i} \mid y\right)=\sum_{j=1}^{2} \lambda\left(\alpha_{i} \mid x_{j}\right) P\left(x_{j} \mid y\right)
$$

where, $x=\left\{x_{j=1,2}\right\}$ is a class set and 1 and 2 denote a label for the track and non-track region, respectively, given a set of measurements $y \cdot \alpha=\left\{\alpha_{i=1,2}\right\}$ is a set of possible actions in determining $x . \lambda()$ is the loss for the actions. $P\left(x_{j} \mid y\right)$ is the posterior probability to be estimated and proportional to the multiplication of likelihood $P\left(y \mid x_{j}\right)$ and prior probability $P\left(x_{j}\right)$. In here, we use training data to learn Gaussian parameters and prior information for determining $P\left(x_{j} \mid y\right)$. As a track is a static object and shows the similarity of geometric configuration along the railway corridor, many training data sets might be not required for this study. Thus, one strip is randomly selected in the long railway corridor area and used for the training process. In the case of Bayesian decision rule for two categories like this study, the likelihood ratio can be formulated as shown in Eq. (3) and the corresponding class label is assigned to each search window. The loss $\lambda()$ is normally quantified based on $0-1$ loss function due to complexity reasons (Schluter et al., 2005). That is, $\lambda()$ satisfies $\lambda()=0$ if $i=j$, otherwise, $\lambda()=1$.

$$
\frac{P\left(y \mid x_{1}\right)}{P\left(y \mid x_{2}\right)}>\frac{\lambda_{12}-\lambda_{22}}{\lambda_{21}-\lambda_{11}} \frac{P\left(x_{2}\right)}{P\left(x_{1}\right)}, \text { if } R\left(\alpha_{1} \mid y\right)<R\left(\alpha_{2} \mid y\right)
$$

Figure 4 shows the example of the railway candidate track regions and the selected main track region minimizing its expected loss.

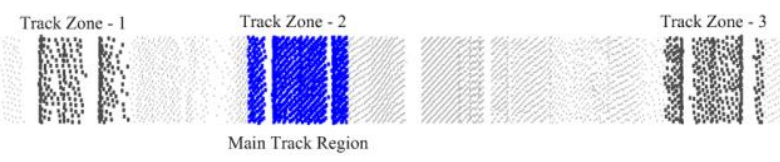

Figure 4. Potential railway track zone detection (dark gray color) and the main track region determination (blue color) in the initial strip.

2.2.3 Initialization of track models: Once track candidate points are detected in the previous step, rail head points representing a track surface are required to be segmented from the track points for the use of reconstructing 3D track models. In this study, the mean shift clustering using a flat kernel is considered as an effective method in grouping rail head points. That is, as the spatial distribution of track points shows the combination of different geometric patterns (i.e., horizontal segment for rail head and vertical segment for rail web) with respect to the height direction (Figure 3(c)), the region growing technique can be easy to be applied and gather points representing the track surface using closeness property between points. The $3 \mathrm{D}$ track model is reconstructed using the $3^{\text {rd }}$ polynomial model in the form: 


$$
R=\left\{[x, y, z]^{T} \mid x=f_{H}(y), z=f_{V}(y)\right\}
$$

where, $f(y)=c_{0}+c_{1} y+c_{2} y^{2}+c_{3} y^{3}$ defines the $3^{\text {rd }}$ polynomial model and $\mathrm{H}$ and $\mathrm{V}$ indicate the horizontal and vertical plane, respectively. $[x, y, z]^{T}$ is defined in the local coordinate frame in which the y-coordinate follows the track vector.

Figure 5 depicts the example of the track component clustering and track modeling. The process starts with the track region detected in the first strip (Figure 5(a)). Next, track points are detected based on the GMM-EM algorithm from the track region (Figure 5(b)). The track points are then segmented into rail head and web patches using the mean shift clustering (Figure 5(c)). Finally, 3D track models are reconstructed using rail head points (Figure 5(d)).

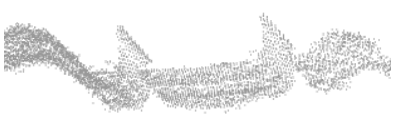

(a)

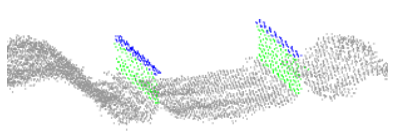

(c)

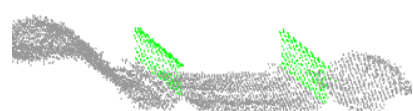

(b)

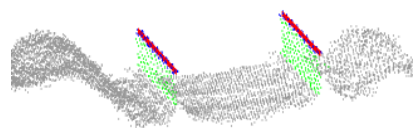

(d)
Figure 5. (a) Main track region, (b) track candidate points (green color), (c) rail head point clustering (blue color), and (d) $3 \mathrm{D}$ track model reconstruction (red color).

\subsection{Kalman Filter-based Railway Tracking}

The Kalman filter is known as a best linear estimator and recursive filter (Kalman, 1960) and is widely used in many realtime applications including navigation, object tracking as well as economics (Faragher, 2012). That is, it is designed to estimate optimal parameters of interest from noisy measurements by minimizing the mean square error and improve the estimation from a series of measurements observed over time. In this study, as parameters used for the track tracking is not associated with a time epoch, a distance comprising a space along a track is used as the time variable. The proposed railway tracking procedure based on the Kalman filter framework is composed of two steps: the space update and the measurement update. The used state vector consists of two parts: track end point $\left[T_{x} T_{y}\right]^{T}$ and $3^{\text {rd }}$ polynomial model parameters for horizontal and vertical plane $\left[M_{H} M_{V}\right]^{T}$. In the space update, the track end positions at the current space $(k)$ is predicted by Eq. (5) using the state vector at the previous space $(k-1)$.

$$
\left[\begin{array}{c}
T_{x} \\
T_{y}
\end{array}\right]_{k}=\left[\begin{array}{c}
T_{x} \\
T_{y}
\end{array}\right]_{k-1}+\Delta L\left[\begin{array}{c}
\sin \theta \\
\cos \theta
\end{array}\right]
$$

where, $\Delta L$ is a distance along the track vector and $\theta$ denotes the orientation of the track vector. In this study, the $\Delta L$ is dynamically determined by measuring deviation between a linear and non-linear track vector under the assumption that a track has a linear property in the local search area. Thus, the track end point is specified along the track vector at the position with the deviation which is more than the pre-determined tolerance.

After the space update, a potential track region is determined using $\Delta L$ and rail head points are segmented from track points detected based on the GMM-EM technique and mean shift clustering. The state vector is then estimated using the track head points detected at the current space $(\mathrm{k})$ based on the measurement update step of the Kalman filter.

Figure 6 presents the entire process of Kalman filtering for 3D track modelling and railway tracking in the selected track trajectory. The process started with the results of the railway track localization in the first strip.

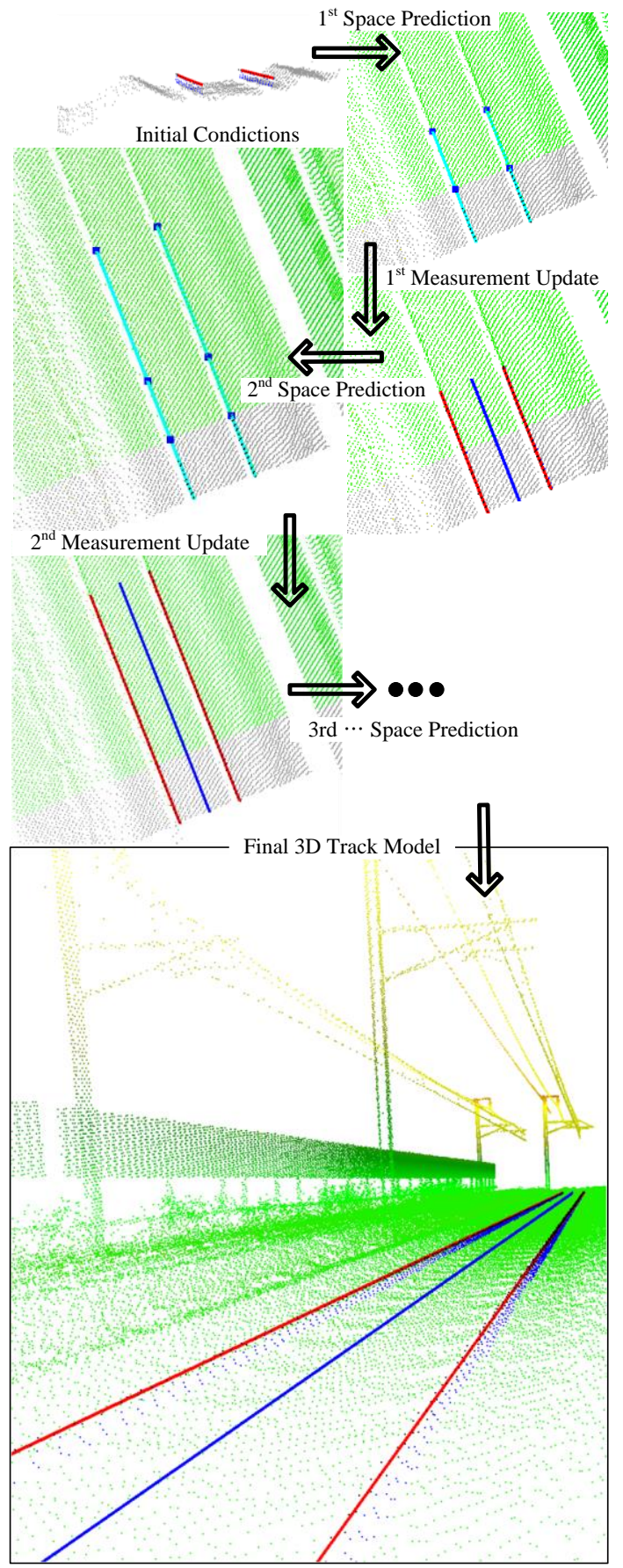

Figure 6. Kalman filter-based railway tracking procedure for detecting track points (blue color), 3D track model (red color), and track center line (blue color). 


\section{EXPERIMENTAL RESULT}

The performance of the proposed method was evaluated with mobile LiDAR data containing two double-tracks (i.e., four single track pairs) which is located between Uiwang and Cheonan near Seoul of South Korea. The laser point data are acquired from the Optech's Lynx M1 mobile mapping system mounted on a vehicle and installed on a train wagon with a speed of $60 \mathrm{~km} / \mathrm{h}$. The mobile mapping system is able to collect up to $1,000,000$ points per second in the maximum range of 200 $m$ with $20 \%$ reflectivity. The relative and absolute accuracy of points are reported to be less than $1 \mathrm{~cm}$ and $5 \mathrm{~cm}$, respectively. Figure 7 shows the test data covering about $100 \mathrm{~m}$ (length) $\times$ $50 \mathrm{~m}$ (width) in which mobile laser points are colorized from blue to red colour according to their height. Approximately 0.4 million laser points at an average point density of 85 (points $/ \mathrm{m}^{2}$ ) were collected.

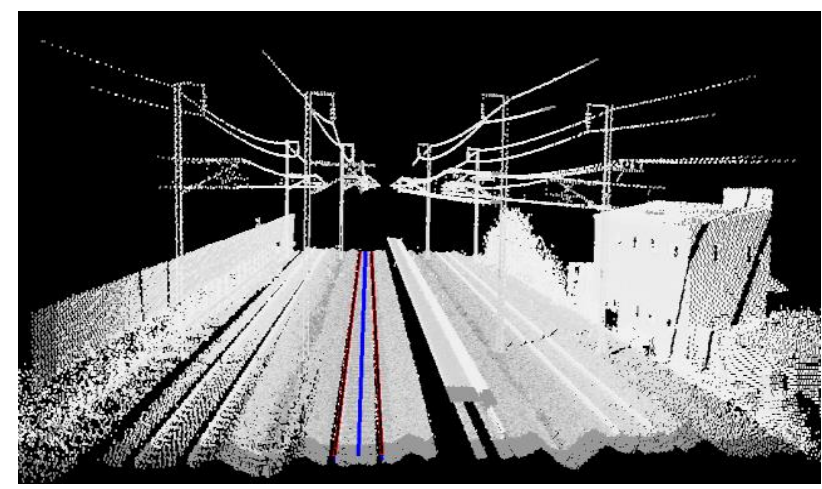

Figure 7. Raw mobile LiDAR data set colorized from black to white w.r.t. the intensity strength of point clouds, and 3D railway track models (red color) and track center line (blue color) in the main track region.

We evaluated the detection rate of track points by comparing the results with reference. In here, the classification was manually conducted using the commercial software (TerraScan of Terrasolid) to produce the reference data. The detection rate was measured by two criteria, completeness and correctness, by counting three factors: TP (True Positive), FN (False Negatives), and FP (False Positives) (Jwa and Sonh, 2012). A total number of 8621 points were classified as track points in the reference data. As we compared the reference data to the track detection results, 7074 of TP, 1574 of FN, and 7 of FP are counted. This leads that the completeness was calculated as $81.7 \%$ and the correctness was measured as $99.9 \%$. As shown in the detection results, the proposed method for detecting track points intends to produce a higher rate of correctness by producing fewer FP, while more FN leads to lower rate of completeness. The main reason is that points representing the rail foot were also labelled as track points in the reference, while the detection results mostly contains points representing the rail head and web for track points. We believe that the performance of track modelling is not affected by the relatively low completeness rate because 3D track models are reconstructed using rail head points. The track modelling results are shown as thick red lines in Figure 7. The track modelling accuracy (RMSE) measured by the discrepancy between track models and the corresponding track points, resulting in the accuracy of $1.79 \mathrm{~cm}$ in $\mathrm{X}-\mathrm{Y}$ plane. The modelling accuracy can be considered as a reasonable result by taking into account the point accuracy of less than 5 $\mathrm{cm}$ and the width of rail head of approximately $6.5 \mathrm{~cm}$.
In addition, geometric information on railway tracks can be extracted as by-products which become important factors in performing the precise railway risk management. As shown in Figure 8, the extracted track models show the variation of track gauge with the mean of $1.5 \mathrm{~m}$ and its standard deviation of 0.11 $\mathrm{cm}$ (Figure 8(a)) and the variation of height difference of $1.8 \mathrm{~cm}$ and its standard deviation of $0.21 \mathrm{~cm}$ (Figure $8(\mathrm{~b})$ ) for the test data.

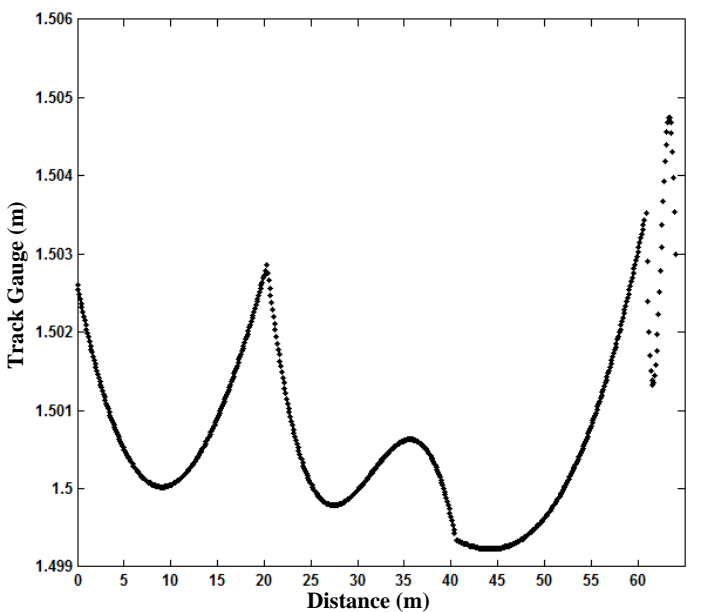

(a)

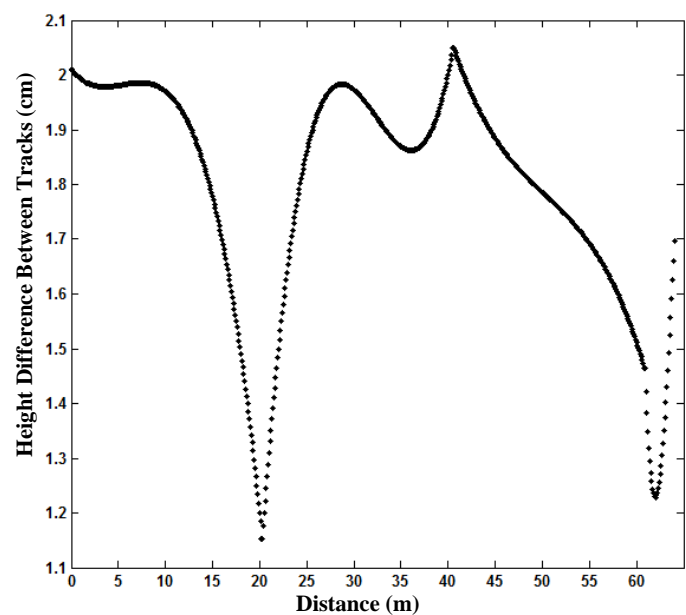

(b)

Figure 8: Geometric information of tracks derived from 3D track models reconstructed: (a) variation of track gauge and (b) variation of height difference of a pair of single tracks.

\section{CONCLUSION}

Geometric modelling of railway tracks has become an issue of increasing importance for the effective and precise railway track monitoring. This is due to the fact that track models are regarded as an essential component for identifying potentially dangerous situations such as inelastic deformations of the steel tracks. This study addressed the new method, Kalman filterbased railway tracking, for detecting railway track points and reconstructing 3D railway track models from mobile LiDAR data. The proposed method provided the accurate detection rate of railway track points with the completeness of $82 \%$ and the correctness of $99 \%$. The resulting track models are good with the RMSE of $1.8 \mathrm{~cm}$, which means that those models are fitted well to the corresponding track points. Consequently, in the 
railway risk management, those high modelling quality can provide a great benefits to perform a rapid and correct the assessed clearance quantity in the railway corridor area. As future work, to improve the robustness of proposed method, the recognition process of various track related objects such as turnouts and switch stand is required to be developed.

\section{ACKNOWLEDGEMENTS}

This research was supported by a grant (15RTRP-B067297-03) from Railroad Technology Research Program (Technology development on the positioning detection of railroad with high precision) funded by Ministry of Land, Infrastructure and Transport of Korean government.

\section{REFERENCES}

Beger, R., Neubert, M., Herold, H., 2012. Towards adaptive image processing-automated extraction of railroad tracks from extremely high resolution aerial imagery. Proceedings of the $4^{\text {th }}$ GEOBIA, 7-9 May, pp. 536-540.

Bishop, C. M., 2006. Pattern recognition and machine learning. Springer, New York.

Cho, W., Jwa, Y., Chang, H., Lee, S., 2004. Pseudo-grid based building extraction using airborne LiDAR data. IAPRS, 35(B3), pp. 378-383.

Dalal N., Triggs B., 2005. Histograms of oriented gradients for human detection. CVPR, pp. 886-893.

Espino, J., C., Stanciulescu, B., 2012. Rail extraction technique using gradient information and a priori shape model. $15^{\text {th }}$ International IEEE Conference on Intelligent Transportation System, Anchorage, Alaska, USA, 16-19 Sep., pp. 1132-1136.

Faragher, R., 2012. Understanding the basis of the Kalman filter via a simple and intuitive derivation. IEEE Signal Process, Mag., 29(5), pp. 128-132.

Jwa, Y., Sonh, G., 2012. A piecewise catenary curve model growing for 3D power line reconstruction. PE\&RS, 78(12), pp. $1227-1240$

Kalman, R. E., 1960. A new approach to linear filtering and prediction problem. J. Basic Eng., 82(1), pp. 35-45.

Muhamad, M., Kusevic, K., Mrstik, P;, Greenspan, M., 2013. Automatic rail extraction in terrestrial and airborne LiDAR data, International Conference on 3D Vision, pp. 303-309.

Oude Elberink, S., Khoshelham, K., Arastounia, M., Diaz Benito, D., 2013. Rail track detection and modelling in mobile laser scanner data. ISPRS Annals of the Photogrammetry Remote Sensing and Spatial Information Sciences, II-5/W2, pp. 223-228.

Ozerov, A., Lagrange, M., and Vincent, E., 2011. GMM-based classification from noisy features. 1st Int. Workshop on Machine Listening in Multisource Environments, Florence, Italy, pp. 30-35.
Qi, Z., Tian, Y., Shi, Y., 2013. Efficient railway tracks detection and turnouts recognition method using HOG features, Neural Comput \&Applic., 23, pp. 245-254.

Resendiz, E., Hart, J. M., Ahuia, N., 2013. Automated visual inspection of railroad tracks, IEEE Transactions On Intelligent Transportation Systems, 14(2), pp. 751-760.

Schluter, R., Scharrenbach, T., Steinbill, V., Ney, H., 2005. Bayes risk minimization using metric loss functions. In Proceedings of the European Conference on Speech Communication and Technology, Interspeech, Portugal, September, pp. 1449-1452.

Sohn, G., Dowman, I., 2007. Data fusion of high-resolution satellite imagery and LiDAR data for automatic building extraction. ISPRS Journal of Photogrammetry and Remote Sensing, 62 (1), 43-63.

Yang, B., Fang, L., 2014. Automated extraction of 3D railway tracks from mobile laser scanning point clouds. IEEE Journal of Selected Topics In Applied Earth Observations and Remote Sensing, pp. 4750-4761. 